\title{
Smooth Extensions of Bernoulli Shifts
}

by

\author{
Zbigniew S. KOWALSKI
}

Presented by Andrzej LASOTA

Summary. For homographic extensions of the one-sided Bernoulli shift we construct $\sigma$-finite invariant and ergodic product measures. We apply the above to the description of invariant product probability measures for smooth extensions of one-sided Bernoulli shifts.

0. Introduction. Let $\sigma$ be the one-sided $(p, q)$-Bernoulli shift on the space $\Omega=\{0,1\}^{\mathbb{N}}, \mathbb{N}=\{0,1,2, \ldots\}$, with the $(p, q)$-measure $\mu_{p}$ on $(\Omega, \mathcal{B})$, where $\mathcal{B}$ is the Borel product $\sigma$-algebra. Let us consider two transformations $T_{0}, T_{1}$ of the interval $[0,1]$ onto itself such that $T_{i} \in C^{2}[0,1], T_{i}^{\prime}>0$, $T_{i}(0)=0, T_{i}(1)=1$ for $i=0,1$ and $T_{0} \geq I, T_{1} \leq I$ where $I(x)=x$ for $x \in[0,1]$. We define the transformation

$$
T(\omega, x)=\left(\sigma(\omega), T_{\omega(0)}^{-1}(x)\right) .
$$

This transformation is the realization of the random map $T(x)=T_{0}^{-1}(x)$ with probability $p$ and $T(x)=T_{1}^{-1}(x)$ with probability $q$. Let $\Lambda$ denote the Lebesgue measure on $[0,1]$. It will cause no confusion if we use the same letter to designate the Lebesgue measure on $\mathbb{R}^{+}$. Let $M_{p}$ denote the set of $T$-invariant measures such that $m \mid \mathcal{B} \times[0,1]=\mu_{p}$ for $m \in M_{p}$. The product measures in $M_{p}$ allow us to describe the distribution of almost every trajectory of random map. Therefore our purpose is to get a description of such measures in $M_{p}$. Some results on this topic have been obtained in [K] for transformations $T_{i}=\left(1-\varepsilon_{i}\right) x+\varepsilon_{i} g(x), i=0,1$, where $g \in C^{2}[0,1], g(0)=0$, $g(1)=1,\left(1-\sup g^{\prime}\right)^{-1}<\varepsilon_{0}, \varepsilon_{1}<\left(1-\inf g^{\prime}\right)^{-1}$. Here we additionally assume that there exists exactly one point $x_{0}$ for which $g^{\prime}\left(x_{0}\right)=1$ and

2000 Mathematics Subject Classification: Primary 37A40.

Key words and phrases: homographic extension of one-sided Bernoulli shift, invariant product measure.

Research supported by KBN Grant 2 P03A 04622 (2002-2005). 
either $g^{\prime}(x)<1$ for $x<x_{0}$ or $g^{\prime}(x)>1$ for $x<x_{0}$. Furthermore, the above mentioned paper contains conditions which ensure that $M_{p}$ consists of product measures and that it contains no absolutely continuous measures.

The present paper extends these results as follows. In Section 1 we consider commuting homographic transformations $T_{i}, i=0,1$. For the homographic extensions of the one-sided Bernoulli shift we determine $\sigma$-finite invariant and ergodic equivalent product measures. Next, we describe the cluster points of $\left(\mathcal{A}^{n} I\right)_{n \in \mathbb{N}}$, where $\mathcal{A}$ is the operator acting on the set of distribution functions of probability measures determined by $p$ and $T_{i}, i=0,1$. In Section 2 we consider the transformations $T_{i}$ (the same as in $[\mathrm{K}]$ ), $i=0,1$, and the operator $\mathcal{A}_{T}$ determined by $p$ and $T_{i}, i=0,1$. We prove (Theorem 3 ) that if $\lim _{n \rightarrow \infty} \mathcal{A}_{T}^{n} I=0$ or 1 then the set of product measures in $M_{p}$ is $\operatorname{conv}\left\{\mu_{p} \times \delta_{\{0\}}, \mu_{p} \times \delta_{\{1\}}\right\}$. Next, using the results of Section 1 we show (for some instances) how the cluster points of $\left(\mathcal{A}_{T}^{n} I\right)_{n \in \mathbb{N}}$ may be determined. In particular we extend the description of $M_{p}$ for the example from $[\mathrm{K}]$.

1. Homographic extensions of Bernoulli shifts. Let us consider the transformation of the unit interval of the form

$$
T(x)=\frac{a x+b}{c x+d} \quad \text { where } a, b, c, d \in \mathbb{R} .
$$

Under the assumptions:

$$
\begin{array}{cl}
T:[0,1] \rightarrow[0,1], & T(0)=0, \quad T(1)=1, \\
T^{\prime}(0)>1, T^{\prime}(1)<1 & \text { or } \quad T^{\prime}(0)<1, T^{\prime}(1)>1
\end{array}
$$

we have

$$
T(x)=T_{\lambda}(x)=\frac{x}{\lambda x+1-\lambda}, \quad \text { where } \quad \lambda \in(-\infty, 1) \backslash\{0\} .
$$

Moreover, $T_{\lambda} \geq I$ for $\lambda \in(0,1)$ and $T_{\lambda} \leq I$ for $\lambda \in(-\infty, 0)$. The oneparameter family $T_{\lambda}$ of homographic maps commutes and

$$
T_{\lambda_{0}} \circ T_{\lambda_{1}}=T_{1-\left(1-\lambda_{0}\right)\left(1-\lambda_{1}\right)}, \quad T_{\lambda}^{-1}=T_{-\lambda /(1-\lambda)} .
$$

Let $\sigma$ be the one-sided $(p, q)$-Bernoulli shift. Take $T_{\lambda_{0}}, T_{\lambda_{1}}$ for $\lambda_{0} \in(0,1)$ and $\lambda_{1} \in(-\infty, 0)$ and define the transformation

$$
T(\omega, x)=\left(\sigma(\omega), T_{\lambda_{\omega(0)}}^{-1}(x)\right) .
$$

Let $\mathcal{D}$ be the set of distribution functions of probability measures on $[0,1]$. Define the operator $\mathcal{A}$ on $\mathcal{D}$ as follows:

$$
\mathcal{A} F(x)=p F\left(T_{\lambda_{0}}(x)\right)+q F\left(T_{\lambda_{1}}(x)\right) \quad \text { for } F \in \mathcal{D} .
$$

Let $\nu_{F}$ denote the measure determined by $F$. 
FACT $1([\mathrm{~K}])$. The measure $\mu_{p} \times \nu_{F}$ is T-invariant if and only if $\mathcal{A} F=F$.

We compute $\mathcal{A}^{n} I$ :

$$
\begin{aligned}
\mathcal{A}^{n} I & =\sum_{k=0}^{n}\left(\begin{array}{l}
n \\
k
\end{array}\right) p^{k} q^{n-k} T_{1-\left(1-\lambda_{0}\right)^{k}\left(1-\lambda_{1}\right)^{n-k}} \\
& =\frac{x}{1-x} \sum_{k=0}^{n}\left(\begin{array}{l}
n \\
k
\end{array}\right) p^{k} q^{n-k}\left[\left(1-\lambda_{0}\right)^{k}\left(1-\lambda_{1}\right)^{n-k}+\frac{x}{1-x}\right]^{-1}
\end{aligned}
$$

for $x \in(0,1)$. Consequently,

$$
\mathcal{A}^{n} I(x) \leq \frac{x}{1-x}\left(\frac{p}{1-\lambda_{0}}+\frac{q}{1-\lambda_{1}}\right)^{n} \quad \text { for } x \in(0,1) .
$$

Since

we get

$$
\frac{p}{1-\lambda_{0}}+\frac{q}{1-\lambda_{1}}<1 \Leftrightarrow p<\frac{\lambda_{1}\left(\lambda_{0}-1\right)}{\lambda_{0}-\lambda_{1}}
$$

FACT 2.

$$
\lim _{n \rightarrow \infty} \mathcal{A}^{n} I(x)=0 \quad \text { for } x \in[0,1) \quad \text { and } \quad p<\frac{\lambda_{1}\left(\lambda_{0}-1\right)}{\lambda_{0}-\lambda_{1}} .
$$

FACT 3. $\mathcal{A} I \geq I$ for $p \geq \frac{\lambda_{1}}{\lambda_{1}-\lambda_{0}}$ and $\mathcal{A} I \leq I$ for $p \leq \frac{\lambda_{1}\left(\lambda_{0}-1\right)}{\lambda_{0}-\lambda_{1}}$

Proof. Observe that $\mathcal{A} I \geq I$ if and only if

$$
d(x)=\frac{p}{\lambda_{0} x+1-\lambda_{0}}+\frac{q}{\lambda_{1} x+1-\lambda_{1}} \geq 1
$$

for every $x \in[0,1]$. In particular

$$
d(0) \geq 1 \Leftrightarrow p \geq \frac{\lambda_{1}\left(\lambda_{0}-1\right)}{\lambda_{0}-\lambda_{1}} \text { and } d(1)=1 .
$$

Now $d$ attains its minimum exactly at

$$
x_{0}=1+\left(\sqrt{\frac{-\lambda_{0} p}{\lambda_{1} q}}-1\right)\left(\lambda_{0}-\lambda_{1} \sqrt{\frac{-\lambda_{0} p}{\lambda_{1} q}}\right)^{-1} .
$$

Since $x_{0} \geq 1$ if and only if $p \geq \frac{\lambda_{1}}{\lambda_{1}-\lambda_{0}}$, we get $\mathcal{A} I \geq I$ for $p \geq \frac{\lambda_{1}}{\lambda_{1}-\lambda_{0}}$. The proof of the case $\mathcal{A} I \leq I$ is similar.

We now state a result that will be of use later.

LEMMA 1.

$$
\frac{d}{d x}\left(\mathcal{A}^{n} I\right)(x) \leq \frac{2}{(1-x) x} \quad \text { for } x \in(0,1) \text { and } n \in \mathbb{N} .
$$

Proof. Let

$$
f_{n}(x)=\sum_{k=0}^{n}\left(\begin{array}{l}
n \\
k
\end{array}\right) p^{k} q^{n-k}\left(a^{k} b^{n-k}+x\right)^{-1}
$$


where $a=1-\lambda_{0}, b=1-\lambda_{1}$. Then

$$
f_{n}^{\prime}(x)=-\sum_{k=0}^{n}\left(\begin{array}{l}
n \\
k
\end{array}\right) p^{k} q^{n-k}\left(a^{k} b^{n-k}+x\right)^{-2} .
$$

Therefore

$$
\left|f_{n}^{\prime}(x)\right| \leq \frac{1}{2 x} \sum_{k=0}^{n}\left(\begin{array}{l}
n \\
k
\end{array}\right) p^{k} q^{n-k}\left(a^{k} b^{n-k}+x / 2\right)^{-1}
$$

and consequently

$$
\left|f_{n}^{\prime}(x)\right| \leq \frac{1}{2 x} f_{n}\left(\frac{x}{2}\right) .
$$

Combining the equalities

$$
f_{n}(x)=\frac{1}{x} \mathcal{A}^{n} I\left(\frac{x}{1+x}\right) \quad \text { and } \quad \mathcal{A}^{n} I(x)=\frac{x}{1-x} f_{n}\left(\frac{x}{1-x}\right)
$$

with (3) we get

$$
\frac{d}{d x} \mathcal{A}^{n} I(x)=\frac{1}{(1-x)^{2}} f_{n}\left(\frac{x}{1-x}\right)+\frac{x}{1-x} \frac{1}{(1-x)^{2}} f_{n}^{\prime}\left(\frac{x}{1-x}\right)
$$

and

$$
\frac{d}{d x} \mathcal{A}^{n} I(x) \leq \frac{1}{(1-x) x}\left(\mathcal{A}^{n} I(x)+\mathcal{A}^{n} I\left(\frac{x}{2-x}\right)\right) .
$$

This gives

$$
\frac{d}{d x} \mathcal{A}^{n} I(x) \leq \frac{2}{(1-x) x} \quad \text { for } x \in(0,1)
$$

and the proof is complete.

The next lemma ensures the existence of a $\sigma$-finite $T$-invariant measure.

Lemma 2. For every

$$
p \neq \frac{\ln \left(1-\lambda_{1}\right)}{\ln \left(\frac{1-\lambda_{1}}{1-\lambda_{0}}\right)}
$$

there exists $s \neq 0$ such that

$$
\mathcal{A} F_{p}=F_{p} \quad \text { for } F_{p}=\left(\frac{x}{1-x}\right)^{s} .
$$

Proof. We first observe that the following identity holds:

$$
\frac{T_{\lambda}(x)}{1-T_{\lambda}(x)}=\frac{1}{1-\lambda} \frac{x}{1-x}
$$


for $x \in(0,1)$ and $\lambda \in(-\infty, 1)$. From this it follows that

$$
\begin{aligned}
\mathcal{A}\left(\frac{x}{1-x}\right)^{s} & =p \frac{1}{\left(1-\lambda_{0}\right)^{s}}\left(\frac{x}{1-x}\right)^{s}+q \frac{1}{\left(1-\lambda_{1}\right)^{s}}\left(\frac{x}{1-x}\right)^{s} \\
& =\left[p \frac{1}{\left(1-\lambda_{0}\right)^{s}}+q \frac{1}{\left(1-\lambda_{1}\right)^{s}}\right]\left(\frac{x}{1-x}\right)^{s} .
\end{aligned}
$$

Therefore,

$$
\begin{aligned}
\mathcal{A}\left(\frac{x}{1-x}\right)^{s}=\left(\frac{x}{1-x}\right)^{s} & \Leftrightarrow p \frac{1}{\left(1-\lambda_{0}\right)^{s}}+q \frac{1}{\left(1-\lambda_{1}\right)^{s}}=1 \\
& \Leftrightarrow p=\frac{\left(1-\lambda_{1}\right)^{s}-1}{\left(\frac{1-\lambda_{1}}{1-\lambda_{0}}\right)^{s}-1}
\end{aligned}
$$

Let

$$
\gamma(s)=\frac{\left(1-\lambda_{1}\right)^{s}-1}{\left(\frac{1-\lambda_{1}}{1-\lambda_{0}}\right)^{s}-1} \quad \text { for } s \neq 0
$$

and

$$
\gamma(0)=\lim _{s \rightarrow 0} \gamma(s)=\frac{\ln \left(1-\lambda_{1}\right)}{\ln \left(\frac{1-\lambda_{1}}{1-\lambda_{0}}\right)} .
$$

The function $\gamma$ is continuous, strictly decreasing on $\mathbb{R}$ and $\lim _{s \rightarrow-\infty} \gamma(s)=1$, $\lim _{s \rightarrow \infty} \gamma(s)=0$. This shows that for every $p \neq \gamma(0)$ there exists exactly one $s \neq 0$ such that (4) holds, which completes the proof.

Let $T$ be given by (1) and $m=\mu_{p} \times \nu_{F_{p}}$. By using the fact that $\mu_{p}$ is Bernoulli it is easy to check that

$$
\mu_{p} \times \nu_{F_{p}}\left(T^{-1}(B \times[0, x])\right)=\mu(B) \mathcal{A} F_{p}(x)
$$

for every $B \in \mathcal{B}$ and $x \in[0,1]$. Hence by Lemma 2 we see that $m$ is a $\sigma$-finite infinite $T$-invariant measure equivalent to $\mu_{p} \times \Lambda$. The following theorem ensures the ergodicity of $(T, m)$ :

THEOREM 1. If

$$
\frac{\ln \left(1-\lambda_{1}\right)}{\ln \left(1-\lambda_{0}\right)} \text { is an irrational number and } p \neq \frac{\ln \left(1-\lambda_{1}\right)}{\ln \left(\frac{1-\lambda_{1}}{1-\lambda_{0}}\right)}
$$

then the dynamical system $(T, m)$ is ergodic.

Proof. Let $B$ be a $T$-invariant set. By the theorem of Morita $[\mathrm{M}], B=$ $\Omega \times A$ for a measurable set $A$. Here $T_{i}(A)=A$ for $T_{i}=T_{\lambda_{i}}, i=0,1$. Suppose $\Lambda(A)>0$. Our goal is to show that $\Lambda(A)=1$. To do this we introduce the set $C=J(A)$ where

$$
J(x)=\frac{1-x}{x} .
$$

Since

$$
J\left(T_{0}^{m} T_{1}^{n}(x)\right)=\left(1-\lambda_{0}\right)^{m}\left(1-\lambda_{1}\right)^{n} J(x)
$$


we have

$$
\left(1-\lambda_{0}\right)^{m}\left(1-\lambda_{1}\right)^{n} C=C .
$$

Let $\alpha=\ln \left(1-\lambda_{0}\right) / \ln \left(1-\lambda_{1}\right)$. By the assumption the set $\{m \alpha+n: m, n \in \mathbb{Z}\}$ is dense in $\mathbb{R}$ and consequently the set

$$
E=\left\{\left(1-\lambda_{0}\right)^{m}\left(1-\lambda_{1}\right)^{n}: m, n \in \mathbb{Z}\right\}
$$

is dense in $\mathbb{R}^{+}$. Suppose on the contrary that $\Lambda(A)<1$. Then $\Lambda\left(\mathbb{R}^{+} \backslash C\right)>0$. Let $x$ and $y \neq 0$ be density points of $\mathbb{R}^{+} \backslash C$ and $C$, respectively. For $\varepsilon<1 / 2$ there exists $\delta>0$ such that for any interval $Q$, if $\Lambda(Q)<\delta$ and $x \in Q$ (resp. $y \in Q$ ) then

$$
\Lambda\left(Q \cap \mathbb{R}^{+} \backslash C\right) \geq(1-\varepsilon) \Lambda(Q) \quad(\text { resp. } \Lambda(Q \cap C) \geq(1-\varepsilon) \Lambda(Q)) .
$$

Let $Q$ be such that $y \in Q,(x / y) \Lambda(Q)<\delta$ and $\Lambda(Q)<\delta$. By the density of $E$ in $\mathbb{R}^{+}$there exists $\beta \in E$ such that $\beta \Lambda(Q)<\delta$ and $x \in \beta Q$. Thus we get

$$
\Lambda\left(\beta Q \cap \mathbb{R}^{+} \backslash C\right)>(1-\varepsilon) \beta \Lambda(Q)
$$

and also

$$
\begin{aligned}
\Lambda(\beta Q \cap C) & =\Lambda(\beta Q \cap \beta C)=\beta \Lambda(Q \cap C) \\
& \geq(1-\varepsilon) \beta \Lambda(Q) \quad \text { by }(6),
\end{aligned}
$$

which is impossible.

Corollary 1. If $\lambda_{0}, \lambda_{1}, p$ satisfy (5) then $(T, m)$ has no product absolutely continuous invariant probability measure.

Proof. Suppose on the contrary that such a measure exists. Then $T$ is conservative and ergodic. Hence by the unicity of invariant measure ([A, Th. 1.5.6]) the measure $\nu_{F_{p}}$ is finite, which is impossible.

COROllary 2. If $\lambda_{0}, \lambda_{1}, p$ satisfy (5) then the cluster points of

$$
\left(\frac{1}{n} \sum_{k=0}^{n-1} \mathcal{A}^{k} I\right)_{n \in \mathbb{N}}
$$

are constant functions on $(0,1)$.

Proof. By Lemma 1 the family of functions $\mathcal{A}^{n} I, n \in \mathbb{N}$, is uniquely Lipschitzian on $[\varepsilon, 1-\varepsilon]$ for every $0<\varepsilon<1$. Therefore any cluster point $F^{*}$ of

$$
\left(\frac{1}{n} \sum_{k=0}^{n-1} \mathcal{A}^{n} I\right)_{n \in \mathbb{N}}
$$

is Lipschitzian on $(0,1)$. Hence $\nu_{F^{*}}$ is a convex combination of $\delta_{\{0\}}, \delta_{\{1\}}$ and an absolutely continuous measure. Corollary 1 implies that $F^{*}=$ const on $(0,1)$.

The following theorem describes the convergence of $\left(\mathcal{A}^{n} I\right)_{n \in \mathbb{N}}$. 
Theorem 2. (i) If

$$
p \leq \frac{\lambda_{1}\left(\lambda_{0}-1\right)}{\lambda_{0}-\lambda_{1}}
$$

and $\lambda_{0}, \lambda_{1}, p$ satisfy (5) in the case of equality, then

$$
\lim _{n \rightarrow \infty} \mathcal{A}^{n} I=0 \quad \text { almost uniformly on }[0,1) \text {. }
$$

(ii) If

$$
p \geq \frac{\lambda_{1}}{\lambda_{1}-\lambda_{0}}
$$

and $\lambda_{0}, \lambda_{1}, p$ satisfy (5) in the case of equality, then

$$
\lim _{n \rightarrow \infty} \mathcal{A}^{n} I=1 \quad \text { almost uniformly on }(0,1] .
$$

Proof. (i) The first part of (i) follows from Fact 2. Suppose now $p=$ $\lambda_{1}\left(\lambda_{0}-1\right) /\left(\lambda_{0}-\lambda_{1}\right)$ and $\lambda_{0}, \lambda_{1}, p$ satisfy (5). By Fact $3, \mathcal{A}^{n+1} I \leq \mathcal{A}^{n} I \leq I$ for $n=1,2, \ldots$, and by Lemma $1,\left(\mathcal{A}^{n} I\right)_{n \in \mathbb{N}}$ is uniquely Lipschitzian on $[\varepsilon, 1-\varepsilon]$ for every $0<\varepsilon<1$. Combining these with Corollary 2 we get $\lim _{n \rightarrow \infty} \mathcal{A}^{n} I=0$ on $[0,1)$ almost uniformly.

(ii) We first show a new estimate of $\frac{d}{d x} \mathcal{A}^{n} I$ which is more useful for our aim. Let us compute

$$
\frac{d}{d x} \mathcal{A}^{n} I(x)=\frac{1}{x^{2}} \sum_{k=0}^{n}\left(\begin{array}{l}
n \\
k
\end{array}\right)\left[p\left(1-\lambda_{0}\right)\right]^{k}\left[q\left(1-\lambda_{1}\right)\right]^{n-k} T_{1-\left(1-\lambda_{0}\right)^{k}\left(1-\lambda_{1}\right)^{n-k}}^{2}(x) .
$$

Hence

$$
\frac{d}{d x} \mathcal{A}^{n} I(x) \leq \frac{1}{x^{2}}\left[p\left(1-\lambda_{0}\right)+q\left(1-\lambda_{1}\right)\right]^{n}
$$

for $x \in(0,1]$. Now, if $\lambda_{1} /\left(\lambda_{1}-\lambda_{0}\right)<p$ or equivalently $p\left(1-\lambda_{0}\right)+q\left(1-\lambda_{1}\right)$ $<1$ then $\frac{d}{d x} \mathcal{A}^{n} I(x) \rightarrow 0$ uniformly on $(\varepsilon, 1]$ for every $0<\varepsilon<1$. By Fact 3 , $I \leq \mathcal{A}^{n} I \leq \mathcal{A}^{n+1} I$ for $n=1,2, \ldots$ This and the above imply $\lim _{n \rightarrow \infty} \mathcal{A}^{n} I$ $=1$ almost uniformly on $(0,1]$. Let $p=\lambda_{1} /\left(\lambda_{1}-\lambda_{0}\right)$ and suppose $\lambda_{0}, \lambda_{1}, p$ satisfy (5). Since $\frac{d}{d x} \mathcal{A}^{n} I(x) \leq 1 / x^{2}$ for $x \in(0,1], n=1,2, \ldots,\left(\mathcal{A}^{n} I\right)_{n \in \mathbb{N}}$ is uniquely Lipschitzian on $(\varepsilon, 1]$ for every $0<\varepsilon<1$. Combining this with Corollary 2 we get $\lim _{n \rightarrow \infty} \mathcal{A}^{n} I=1$ on $(0,1]$ almost uniformly.

\section{Product measures for smooth extensions of Bernoulli shifts.} Let us consider two transformations of the unit interval

$$
\begin{aligned}
& T_{0}(x)=\left(1-\varepsilon_{0}\right) x+\varepsilon_{0} g(x), \\
& T_{1}(x)=\left(1+\varepsilon_{1}\right) x-\varepsilon_{1} g(x),
\end{aligned}
$$

where $g \in C^{2}[0,1], g(0)=0, g(1)=1,\left(1-\sup g^{\prime}\right)^{-1}<\varepsilon_{0},-\varepsilon_{1}<$ $\left(1-\inf g^{\prime}\right)^{-1}$. We also suppose that there exists exactly one point $x_{0}$ for 
which $g^{\prime}\left(x_{0}\right)=1$ and either $g^{\prime}(x)<1$ for $x<x_{0}$ or $g^{\prime}(x)>1$ for $x<x_{0}$. Let

$$
\mathcal{A}_{T} F=p F\left(T_{0}\right)+q F\left(T_{1}\right) \quad \text { for } F \in \mathcal{D} .
$$

We are thus led to the following strengthening of Lemma 4 of $[\mathrm{K}]$.

Theorem 3. If $\lim _{n \rightarrow \infty} \mathcal{A}_{T}^{n} I=0$ (resp. $\left.\lim _{n \rightarrow \infty} \mathcal{A}^{n} I=1\right)$ for $x \in[0,1)$ (resp. $x \in(0,1])$ then the set of product measures in $M_{p}$ is $\operatorname{conv}\left\{\mu_{p} \times \delta_{\{0\}}\right.$, $\left.\mu_{p} \times \delta_{\{1\}}\right\}$.

We will give the proof only for the case $\lim _{n \rightarrow \infty} \mathcal{A}^{n} I=0$, the second case is similar. We precede the proof by a lemma.

Lemma 3. If $\lim _{n \rightarrow \infty} \mathcal{A}_{T}^{n} I=0$ for $x \in[0,1)$ then for any $G \in C[0,1]$ we have $\lim _{n \rightarrow \infty} \mathcal{A}_{T}^{n} G=G(0)$.

Proof. We first observe that $\lim _{n \rightarrow \infty} \mathcal{A}_{T}^{n}\left(x^{k}\right)=0$ on $[0,1)$ for $k=$ $1,2, \ldots$ This is an immediate consequence of the following implication:

$$
x^{k} \leq x \Rightarrow A_{T}^{n}\left(x^{k}\right) \leq \mathcal{A}_{T}^{n} I \quad \text { for } x \in[0,1] \text { and } n=1,2, \ldots .
$$

Hence, $\lim _{n \rightarrow \infty} \mathcal{A}_{T}^{n} w=w(0)$ on $[0,1)$ for any polynomial $w$. Since $\mathcal{A}_{T}$ is a contraction in the supremum norm and the set of polynomials is dense in $C[0,1]$, we get

$$
\lim _{n \rightarrow \infty} \mathcal{A}_{T}^{n} G=G(0) \quad \text { in }[0,1)
$$

for any $G \in C[0,1]$.

Proof of Theorem 3. Let $G$ be a distribution function. We define two new ones as follows:

$$
G_{1}(x)= \begin{cases}G(x) & \text { for } x \in[0,1) \\ G\left(1^{-}\right) & \text {for } x=1\end{cases}
$$

and $G_{2}(x)=G_{1}\left(x^{-}\right)$for $x \in(0,1]$. Here $G_{1}\left(x^{-}\right)$means the left-hand limit of $G_{1}$ at $x$. Now $G_{1}$ is upper semicontinuous, being nondecreasing and rightcontinuous. Similarly $G_{2}$ is lower semicontinuous. By the definition

$$
G_{2}(x) \leq G(x) \leq G_{1}(x) \quad \text { for } x \in[0,1) .
$$

By Baire's theorem there are sequences of continuous functions $\left(H_{n}^{2}\right)_{n \in \mathbb{N}}$, $\left(H_{n}^{1}\right)_{n \in \mathbb{N}}$ such that $H_{n}^{2} \nearrow G_{2}$ and $H_{n}^{1} \searrow G_{1}$. Also,

$$
\lim _{n \rightarrow \infty} H_{n}^{2}(0)=G_{2}(0)=G(0)=G_{1}(0)=\lim _{n \rightarrow \infty} H_{n}^{1}(0) .
$$

From Lemma 3 and (7) we conclude that

$$
\lim _{n \rightarrow \infty} \mathcal{A}_{T}^{n} G=G(0) \quad \text { for } x \in[0,1) .
$$

Hence, if $\mathcal{A}_{T} G=G$ for $G \in \mathcal{D}$ then $G \equiv G(0)$ on $[0,1)$, which proves the theorem.

To make use of the above theorem we apply Theorem 2 to determine the limit of the sequence $\left(\mathcal{A}_{T}^{n} I\right)_{n \in \mathbb{N}}$. 
EXAMPLE 1. Let $g(x)=T_{\lambda_{0}}(x)$ for $\lambda_{0} \in(0,1)$ and

$$
T_{\varepsilon}(x)=(1+\varepsilon) x-\varepsilon T_{\lambda_{0}}(x) \quad \text { for } 0<\varepsilon<1 / \lambda_{0}-1 .
$$

Put $\mathcal{A}_{\varepsilon}=\mathcal{A}_{T}$. We will determine $\lambda_{1} \in(-\infty, 0)$ such that $\operatorname{sgn}\left(T_{\varepsilon}(x)-\right.$ $\left.T_{\lambda_{1}}(x)\right)=$ const for $x \in(0,1)$ or equivalently

$$
\operatorname{sgn}\left[(x-1)\left(\frac{\lambda_{1}}{\lambda_{1} x+1-\lambda_{1}}+\frac{\varepsilon \lambda_{0}}{\lambda_{0} x+1-\lambda_{0}}\right)\right]=\mathrm{const}
$$

for $x \in(0,1)$. We have

$$
\begin{aligned}
& T_{\varepsilon}(x) \geq T_{\lambda_{1}}(x) \Leftrightarrow \lambda_{1} \leq \frac{\varepsilon \lambda_{0}}{\lambda_{0}(1+\varepsilon)-1}, \\
& T_{\varepsilon}(x) \leq T_{\lambda_{1}}(x) \Leftrightarrow-\varepsilon \lambda_{0} \leq \lambda_{1}<0 .
\end{aligned}
$$

By (8), $\mathcal{A}^{n} I \leq \mathcal{A}_{\varepsilon}^{n} I$ for $n \in \mathbb{N}$ and $\lambda_{1} \leq \varepsilon \lambda_{0} /\left(\lambda_{0}(1+\varepsilon)-1\right)$. Therefore, by Theorem 2(ii),

$$
\lim _{n \rightarrow \infty} \mathcal{A}_{\varepsilon}^{n} I=1 \quad \text { for } p \geq \frac{\varepsilon}{1+\varepsilon} \frac{1}{1-\lambda_{0}} .
$$

If $p=\frac{\varepsilon}{1+\varepsilon} \frac{1}{1-\lambda_{0}}$ then we assume that $p, \lambda_{0}, \lambda_{1}=\varepsilon \lambda_{0} /\left(\lambda_{0}(1+\varepsilon)-1\right)$ satisfy (5). Here we use the fact that

$$
\min \left\{\frac{\lambda_{1}}{\lambda_{1}-\lambda_{0}}: \lambda_{1} \leq \frac{\varepsilon \lambda_{0}}{\lambda_{0}(1+\varepsilon)-1}\right\}
$$

is achieved for $\lambda_{1}=\varepsilon \lambda_{0} /\left(\lambda_{0}(1+\varepsilon)-1\right)$.

REMARK. We have $T_{\varepsilon}(x) \geq T_{\lambda_{0}}^{-1}(x)$ for $\varepsilon \leq 1-\lambda_{0}$ because $T_{\lambda_{0}}^{-1}=$ $T_{-\lambda_{0} /\left(1-\lambda_{0}\right)}$. Hence, by Theorem 2 of $[\mathrm{K}]$,

$$
\lim _{n \rightarrow \infty} \mathcal{A}_{\varepsilon}^{n} I=1 \quad \text { for } p>\max \left\{\frac{1}{2}, \frac{\varepsilon}{1+\varepsilon}\right\} \text { and } \varepsilon \leq 1-\lambda_{0} .
$$

By (9), $\mathcal{A}_{\varepsilon}^{n} I \leq \mathcal{A}^{n} I$ for $n \in \mathbb{N}$ and $-\varepsilon \lambda_{0} \leq \lambda_{1}<0$. Therefore, by Theorem 2(i),

$$
\lim _{n \rightarrow \infty} \mathcal{A}_{\varepsilon}^{n} I=0 \quad \text { for } p \leq \frac{\varepsilon\left(1-\lambda_{0}\right)}{1+\varepsilon} .
$$

If $p=\varepsilon\left(1-\lambda_{0}\right) /(1+\varepsilon)$ then we furthermore assume that $p, \lambda_{0}, \lambda_{1}=-\varepsilon \lambda_{0}$ satisfy (5). Here we use the fact that

$$
\max \left\{\frac{\lambda_{1}\left(\lambda_{0}-1\right)}{\lambda_{0}-\lambda_{1}}:-\varepsilon \lambda_{0} \leq \lambda_{1} \leq 0\right\}
$$

is achieved for $\lambda_{1}=-\varepsilon \lambda_{0}$.

REMARK. We have $T_{\varepsilon}(x) \leq T_{\lambda_{0}}^{-1}(x)$ for $\varepsilon \geq 1 /\left(1-\lambda_{0}\right)$. Hence, by $[\mathrm{K}]$,

$$
\lim _{n \rightarrow \infty} \mathcal{A}_{\varepsilon}^{n} I=0 \quad \text { for } p<\min \left\{\frac{1}{2}, \frac{\varepsilon}{1+\varepsilon}\right\} \text { and } \varepsilon \geq \frac{1}{1-\lambda_{0}} .
$$

We summarize these considerations in the following theorem. 
Theorem 4. If

$$
p \in\left(0, \frac{\varepsilon}{1+\varepsilon}\left(1-\lambda_{0}\right)\right] \cup\left[\frac{\varepsilon}{1+\varepsilon} \frac{1}{1-\lambda_{0}}, 1\right)
$$

then the set of product measures in $M_{p}$ is

$$
\operatorname{conv}\left\{\mu_{p} \times \delta_{\{0\}}, \mu_{p} \times \delta_{\{1\}}\right\} .
$$

Here

$$
p= \begin{cases}\frac{\varepsilon}{1+\varepsilon}\left(1-\lambda_{0}\right) & \text { if } p, \lambda_{0}, \lambda_{1}=-\varepsilon \lambda_{0} \text { satisfy }(5), \\ \frac{\varepsilon}{1+\varepsilon} \frac{1}{1-\lambda_{0}} & \text { if } p, \lambda_{0}, \lambda_{1}=\frac{\varepsilon \lambda_{0}}{\lambda_{0}(1+\varepsilon)-1} \text { satisfy }(5) .\end{cases}
$$

For $p=\varepsilon /(1+\varepsilon)$,

$$
M_{p}=\operatorname{conv}\left\{\mu_{p} \times \delta_{\{0\}}, \mu_{p} \times \delta_{\{1\}}, \mu_{p} \times \Lambda\right\} .
$$

EXAmPle 2. Let $g(x)=x^{2}$ and

$$
T_{0}(x)=\left(1+\varepsilon_{0}\right) x-\varepsilon_{0} x^{2}, \quad T_{1}(x)=\left(1-\varepsilon_{1}\right) x+\varepsilon_{1} x^{2},
$$

for $0 \leq \varepsilon_{i} \leq 1, i=0,1$. We will determine $\lambda_{0} \in(0,1)$ such that

$$
\operatorname{sgn}\left(T_{0}(x)-T_{\lambda_{0}}(x)\right)=\mathrm{const} \quad \text { for } x \in(0,1)
$$

or equivalently

$$
\operatorname{sgn}\left[(1-x)\left(\varepsilon_{0} \lambda_{0} x-\varepsilon_{0} \lambda_{0}+\varepsilon_{0}-\lambda_{0}\right)\right]=\text { const. }
$$

We have

$$
\begin{aligned}
& T_{0}(x) \geq T_{\lambda_{0}}(x) \Leftrightarrow \lambda_{0} \leq \frac{\varepsilon_{0}}{1+\varepsilon_{0}}, \\
& T_{0}(x) \leq T_{\lambda_{0}}(x) \Leftrightarrow \varepsilon_{0} \leq \lambda_{0} .
\end{aligned}
$$

Similarly,

$$
\operatorname{sgn}\left(T_{1}(x)-T_{\lambda_{1}}(x)\right)=\mathrm{const} \quad \text { for } x \in(0,1)
$$

if and only if

$$
\operatorname{sgn}\left[(x-1)\left(\varepsilon_{1} \lambda_{1} x-\varepsilon_{1} \lambda_{1}+\varepsilon_{1}+\lambda_{1}\right)\right]=\text { const. }
$$

Hence

$$
\begin{aligned}
& T_{1}(x) \geq T_{\lambda_{1}}(x) \Leftrightarrow \lambda_{1} \leq-\frac{\varepsilon_{1}}{1-\varepsilon_{1}}, \\
& T_{1}(x) \leq T_{\lambda_{1}}(x) \Leftrightarrow-\varepsilon_{1} \leq \lambda_{1} .
\end{aligned}
$$

Denote by $\mathcal{A}_{T}$ the operator determined by $p$ and $T_{i}, i=0,1$. By (11) and $(13), \mathcal{A}_{T}^{n} I \leq \mathcal{A}^{n} I$ for $n \in \mathbb{N}$ and for $\left(\lambda_{0}, \lambda_{1}\right) \in\left[\varepsilon_{0}, 1\right] \times\left[-\varepsilon_{1}, 0\right]$. Therefore, by Theorem $2(\mathrm{i})$,

$$
\lim _{n \rightarrow \infty} A_{T}^{n} I=0 \quad \text { for } p \leq \varepsilon_{1} \frac{1-\varepsilon_{0}}{\varepsilon_{0}+\varepsilon_{1}} .
$$


If $p=\varepsilon_{1} \frac{1-\varepsilon_{0}}{\varepsilon_{0}+\varepsilon_{1}}$ then we additionally assume that $p, \lambda_{0}=\varepsilon_{0}, \lambda_{1}=-\varepsilon_{1}$ satisfy (5). Here we use the fact that

$$
\max \left\{\frac{\lambda_{1}\left(\lambda_{0}-1\right)}{\lambda_{0}-\lambda_{1}}:\left(\lambda_{0}, \lambda_{1}\right) \in\left[\varepsilon_{0}, 1\right] \times\left[-\varepsilon_{1}, 0\right]\right\}
$$

is achieved for $\lambda_{0}=\varepsilon_{0}, \lambda_{1}=-\varepsilon_{1}$.

REMARK. By Theorem 2 of [K], if $T_{0} \circ T_{1} \leq I$ then

$$
\lim _{n \rightarrow \infty} \mathcal{A}^{n} I=0 \quad \text { for } p<\min \left\{\frac{1}{2}, \frac{\varepsilon_{1}}{\varepsilon_{0}+\varepsilon_{1}}\right\} .
$$

The condition $T_{0} \circ T_{1} \leq I$ is not always satisfied, e.g. for $\varepsilon_{0}=3 / 4, \varepsilon_{1}=1$.

If the conditions (10) and (12) hold then

$\mathcal{A}_{T}^{n} I \geq \mathcal{A}^{n} I \quad$ for $n \in \mathbb{N}$ and $\left(\lambda_{0}, \lambda_{1}\right) \in\left[0, \frac{\varepsilon_{0}}{1+\varepsilon_{0}}\right] \times\left(-\infty,-\frac{\varepsilon_{1}}{1-\varepsilon_{1}}\right]$.

Therefore, by Theorem 2(ii),

$$
\lim _{n \rightarrow \infty} \mathcal{A}_{T}^{n} I=1 \quad \text { for } p \geq \varepsilon_{1} \frac{1+\varepsilon_{0}}{\varepsilon_{0}+\varepsilon_{1}} .
$$

If $p=\varepsilon_{1} \frac{1+\varepsilon_{0}}{\varepsilon_{0}+\varepsilon_{1}}$ then we furthermore assume that $p, \lambda_{0}=\frac{\varepsilon_{0}}{1+\varepsilon_{0}}, \lambda_{1}=-\frac{\varepsilon_{1}}{1-\varepsilon_{1}}$ satisfy (5). Here we use the fact that

$$
\min \left\{\frac{\lambda_{1}}{\lambda_{1}-\lambda_{0}}:\left(\lambda_{0}, \lambda_{1}\right) \in\left[0, \frac{\varepsilon_{0}}{1+\varepsilon_{0}}\right] \times\left(-\infty,-\frac{\varepsilon_{1}}{1-\varepsilon_{1}}\right]\right\}
$$

is achieved for $\lambda_{0}=\frac{\varepsilon_{0}}{1+\varepsilon_{0}}, \quad \lambda_{1}=-\frac{\varepsilon_{1}}{1-\varepsilon_{1}}$.

REMARK. By Theorem 2 of [K], if $T_{0} \circ T_{1} \geq I$ then

$$
\lim _{n \rightarrow \infty} \mathcal{A}_{T}^{n} I=1 \quad \text { for } p>\max \left\{\frac{1}{2}, \frac{\varepsilon_{1}}{\varepsilon_{0}+\varepsilon_{1}}\right\} \text {. }
$$

The condition $T_{0} \circ T_{1} \geq I$ is not always satisfied, e.g. for $\varepsilon_{0}=\varepsilon_{1}=1 / 2$.

We summarize Example 2 in the following theorem:

THEOREM 5. If

$$
p \in\left(0, \varepsilon_{1} \frac{1-\varepsilon_{0}}{\varepsilon_{0}+\varepsilon_{1}}\right] \cup\left[\varepsilon_{1} \frac{1+\varepsilon_{0}}{\varepsilon_{0}+\varepsilon_{1}}, 1\right)
$$

then the set of product measures in $M_{p}$ is

$$
\operatorname{conv}\left\{\mu_{p} \times \delta_{\{0\}}, \mu_{p} \times \delta_{\{1\}}\right\} .
$$

Here

$$
p=\left\{\begin{array}{l}
\varepsilon_{1} \frac{1-\varepsilon_{0}}{\varepsilon_{0}+\varepsilon_{1}} \quad \text { if } p, \lambda_{0}=\varepsilon_{0}, \lambda_{1}=-\varepsilon_{1} \text { satisfy }(5) \\
\varepsilon_{1} \frac{1+\varepsilon_{0}}{\varepsilon_{0}+\varepsilon_{1}} \quad \text { if } p, \lambda_{0}=\frac{\varepsilon_{0}}{1+\varepsilon_{0}}, \lambda_{1}=-\frac{\varepsilon_{1}}{1-\varepsilon_{1}} \text { satisfy }(5) .
\end{array}\right.
$$


For $p=\varepsilon_{1} /\left(\varepsilon_{0}+\varepsilon_{1}\right)$ we have

$$
M_{p}=\operatorname{conv}\left\{\mu_{p} \times \delta_{\{0\}}, \mu_{p} \times \delta_{\{1\}}, \mu_{p} \times \Lambda\right\} .
$$

\section{References}

[1] J. Aaronson, An Introduction to Infinite Ergodic Theory, Math. Surveys Monogr. 50, Amer. Math. Soc., 1997, p. 284.

[2] Z. S. Kowalski, Invariant measures for smooth extensions of Bernoulli shifts, Bull. Polish Acad. Sci. Math. 51 (2003), 261-267.

[3] T. Morita, Deterministic version lemmas in ergodic theory of random dynamical systems, Hiroshima Math. J. 18 (1988), 15-29.

Zbigniew S. Kowalski

Institute of Mathematics and Informatics

Wrocław University of Technology

Wybrzeże St. Wyspiańskiego 27

50-370 Wrocław, Poland

E-mail: kowalski@pwr.wroc.pl

Received January 24, 2005;

received in final form May 23, 2005 